\title{
Trends and inequities in amenable mortality between 1997 and 2012 in South Africa
}

\author{
I Neethling, ${ }^{1}$ MSc; P Groenewald, ${ }^{1} \mathrm{MB}$ ChB; H Schneider, ${ }^{2} \mathrm{PhD} ; \mathbf{D}$ Bradshaw, ${ }^{1,3} \mathrm{PhD}$ \\ ${ }^{1}$ Burden of Disease Research Unit, South African Medical Research Council, Cape Town, South Africa \\ ${ }^{2}$ SAMRC Health Services to Systems Research Unit and School of Public Health, Faculty of Community and Health Sciences, \\ University of the Western Cape, Cape Town, South Africa \\ ${ }^{3}$ School of Public Health and Family Medicine, Faculty of Health Sciences, University of Cape Town, South Africa
}

Corresponding author: I Neethling (ineethli@mrc.ac.za)

\begin{abstract}
Background. Amenable mortality comprises causes of death that should not occur with timely and effective healthcare. It is commonly used to assess healthcare performance. It could also be used to assess the effectiveness of the pending National Health Insurance (NHI) in South Africa (SA), but to do this, the level and distribution of amenable mortality are required using a local list of amenable causes.

Objectives. To establish an amenable cause-of-death list appropriate for SA and to determine the levels, trends, geographical distribution, population group differences and international comparisons of mortality amenable to healthcare.

Methods. A local list of amenable causes of death was developed with input from public health and disease-specific medical experts. The Second SA National Burden of Disease estimates were reclassified into amenable mortality. Analyses of age-standardised death rates (ASDRs) and amenable mortality proportions were conducted by province and population group between 1997 and 2012 . Excess mortality in relation to the best-performing province and population group was also analysed. ASDRs for SA were compared with those of European Union (EU) and Organisation for Economic Co-operation and Development (OECD) countries.

Results. The local list of amenable conditions contained 45 causes of death. There were large disparities in amenable mortality between provinces and population groups, which did not attenuate over time. There was an average annual percentage increase in amenable ASDRs, but when HIV/AIDS was excluded from the analysis there was an average annual decrease of $1.12 \%$. In the post-peak HIV/AIDS period between 2008 and 2012, an annual average of 207810 amenable deaths could have been saved if all provinces had the same ASDR as the Western Cape. SA's ASDR was 2.6 and 2.2 times higher than that of the worst-performing EU and OECD country, respectively.

Conclusions. This is the first study known to the authors that has established a local amenable mortality list and described the epidemiology of amenable mortality in SA. Amenable mortality could be used as an indicator of the performance of the pending NHI over time and, in combination with other indicators, could identify areas of the health system that require improvement. Benchmarking could also quantify gaps in health system performance between geographical regions and indicate whether these are reduced with time.
\end{abstract}

S Afr Med J 2019;109(8):597-604. DOI:10.7196/SAMJ.2019.v109i8.13796

South Africa (SA) has high rates of mortality across four broad causes, with disparities among provinces and population groups. ${ }^{[1]}$ The epidemiology of cause of death and area of public health concern, and priority required to address the mortality burden, are well known. ${ }^{[1]}$ However, an indicator of health system performance is required to determine to what extent inadequate healthcare, as opposed to behavioural or socioeconomic factors, is responsible for the high levels of mortality and health disparities. This would also be useful in the context of the United Nations Sustainable Development Goals target $3.8^{[2]}$ to achieve universal health coverage for all by 2030 , and the Lancet Global Health Commission on High Quality Health Systems, which identified that quality of care should improve across conditions and countries. ${ }^{[3]}$

Avoidable mortality was introduced in the 1970s as an indicator of all deaths considered amenable to healthcare, warranting medical audit. ${ }^{[4]}$ The concept has since been applied mostly as an assessment of health system performance at the population level, with the premise that amenable causes of death should not occur in the presence of timely and effective healthcare. ${ }^{[5,6]}$ The introduction of preventable mortality, an indicator of the effectiveness of public health interventions, resulted in a split of avoidable mortality into amenable and preventable causes. ${ }^{[7]}$ Amenable mortality has been used as a metric to assess healthcare performance in population studies - mostly in Europe, New Zealand, Australia and the USA. ${ }^{[5,6,8]}$ The list of amenable causes of death was adapted from the original list by Rutstein et al. ${ }^{[4]}$ to reflect advances in medical technology, as well as differences in expert opinion and changing disease profiles across geographical regions. ${ }^{[7,9,10]}$

Amenable mortality levels have generally decreased faster than preventable and unavoidable mortality levels. ${ }^{[5,11]}$ Populations with poorer socioeconomic status associated with inequities in accessibility and quality of healthcare, have consistently had a higher risk of amenable mortality. ${ }^{[12-14]}$ Evidence suggests that this disparity has been reduced in countries with universal health coverage. ${ }^{[15-17]}$

The concept of amenable mortality is now well established in Europe and Australasia, and the National Health Service in the UK has included it as a summary indicator of treatment outcomes, ${ }^{[18]}$ but there is a dearth of studies assessing amenable mortality in lowand middle-income countries. A recent study, which formed part of the reporting of the Lancet Global Commission on High Quality Health Systems, compared amenable mortality across the globe using mortality data from the 2016 Global Burden of Disease (GBD) study ${ }^{[19]}$ However, GBD mortality estimates have been shown to be inaccurate for $\mathrm{SA},{ }^{[1]}$ and the list of amenable conditions used might 
not be representative of the burden of disease experienced in every country.

$\mathrm{SA}$ is in the process of implementing National Health Insurance (NHI). If this is introduced successfully, amenable mortality could be used as an indicator to track NHI performance in reducing health disparities over time. Amenable mortality is also used to benchmark health system performance between developed countries. ${ }^{[1,20]}$ This approach could be adopted by SA and other developing countries to establish and compare health system performance between countries.

This article reports on research that established an amenable cause-of-death list appropriate for SA, and determined the levels, trends, geographical distribution, population group differences and international comparisons of mortality amenable to healthcare.

\section{Methods}

\section{Study design}

This is a population-based retrospective study of all mortality determined to be amenable to healthcare between 1997 and 2012 in SA.

\section{Data source}

We used the Second SA National Burden of Disease (NBD) study, which includes data from 1997 to 2012, adjusted from Statistics SA (StatsSA) data. The methods are described in detail elsewhere. ${ }^{[21]}$ StatsSA data were adjusted for under-registration of deaths and misclassification of HIV/AIDS deaths, lack of detail on cause of injury, which was estimated from a national sample of mortuaries, and high proportion of ill-defined causes, which were redistributed across valid underlying causes.

The NBD data consist of 140 single causes of death. These were re-categorised into amenable causes of death using an age cut-off of 74 years, because of the difficulty in diagnosing the underlying cause of death in older people due to the high level of comorbidities and following the methods of Nolte and $\mathrm{McKee}^{[5]}$ and Tobias and Yeh. ${ }^{[6]}$ Leukaemia and asthma were restricted to an age limit of 44 years. The rationale is that asthma and chronic obstructive pulmonary disease (COPD) are difficult to distinguish in middle age and older age, while evidence suggests that deaths due to leukaemia are amenable to healthcare between the ages of 15 and 44 years, as death rates for leukaemia have declined substantially between 1960 and 2000 for these age groups. ${ }^{[5,6]}$

\section{Definition and criteria}

The Tobias and Yeh ${ }^{[6]}$ definition of amenable deaths was used, which defines amenable mortality as causes of death that should not occur in the presence of timely and efficient medical care, and can extend life expectancy by $>5$ years in $>50 \%$ of cases after treatment initiation. Amenable causes of death were considered as indicators of secondary prevention or medical care.

\section{Development of an amenable mortality list}

The amenable cause-of-death list used in this study is based on the Nolte and $\mathrm{McKee}^{[5]}$ and Tobias and $\mathrm{Yeh}^{[6]}$ lists, as these were developed through published evidence and expert opinion, and have been extensively used in other studies. ${ }^{[1,22,23]}$

The causes on the two international lists were reviewed by two local public health experts using the Nolte and $\mathrm{McKe}^{[5]}$ and Tobias and $\mathrm{Yeh}^{[6]}$ rationale for inclusion. The list was also presented at a round-table meeting to experts in public health and clinical care. The opinion of disease-specific experts was sought for conditions where there was a difference of opinion, and was then regarded as the final outcome.

\section{Analysis}

Stata IC-13 (StataCorp., USA) and Microsoft Excel 2013 (Microsoft, USA) were used to calculate proportions, age-standardised death rates (ASDRs), mean proportional change in ASDR and excess mortality. The mid-year population estimates by Dorrington ${ }^{[24]}$ were used to calculate ASDRs, and the World Health Organization (WHO) population structure was used for age standardisation. ${ }^{[25]}$

Amenable mortality trends were analysed by age, sex, year, province and population group. Population group was defined according to apartheid classifications (Africans, Whites, Asians and Coloureds). Inequalities in education, income and welfare still persist along racial lines due to the legacy of apartheid. Therefore, population group was analysed as a proxy for socioeconomic status. Trends by population group were analysed only from 2000 onwards owing to the uncertainty of estimates by population groups for the earlier years. HIV/AIDS was also removed from the analysis to determine amenable mortality, as HIV/AIDS conceals trends in other causes.

Furthermore, excess amenable mortality was analysed. This was defined as the number of amenable deaths that could be reduced if the observed population had the same age-specific amenable rates as those of the population with the lowest rates. The analysis was done separately for province and population group. The Western Cape and the white population group had the lowest observed ASDRs between 2008 and 2012. Firstly, expected mortality was calculated by multiplying the ADSR per 100000 population for the province, and population group with the lowest ASDR with the population estimates of each province and population group. Excess mortality was then calculated as the difference in amenable deaths between the observed and expected mortality. Deaths between 2008 and 2012, which represent the post-HIV/AIDS mortality peak, were pooled to attenuate the year-on-year fluctuations in mortality.

An international comparison of amenable ASDRs for SA was done with countries as reported by the Eurostat working group ${ }^{[20]}$ and the Gay et al. ${ }^{[11]}$ study. A direct comparison was made by using the amenable list of conditions and standard populations in these studies.

The 2012 amenable ASDRs for SA were compared with the 2012 estimates of the 33 Eurostat countries using the Eurostat amenable list of causes and the European Union (EU) standard population. Eurostat is a European office that provides statistics to the EU. HIV/AIDS was included in the Eurostat working group study. However, because of the large proportion of HIV/AIDS deaths in the SA data, ${ }^{[1]}$ an alternative comparison was made with the Organisation for Economic Co-operation and Development (OECD) study that excluded HIV/AIDS. ${ }^{[1]}$ For the alternative analysis, the Nolte and McKee ${ }^{[5]}$ and Tobias and $\mathrm{Yeh}^{[6]}$ lists of amenable mortality, together with the OECD 2005 standard population by sex as used by Gay et al., ${ }^{[11]}$ were applied to calculate the ASDR for SA. This was compared with the ASDRs for the 31 OECD countries. ${ }^{[1]}$

\section{Results}

The amenable list for SA comprises 45 causes of death, including 11 communicable causes and 34 non-communicable causes (Table 1). A further categorisation of the causes of death indicates that the list contains 10 different cancer sites, 10 maternal and perinatal causes, 4 cardiovascular causes and 6 causes across respiratory, digestive and genito-urinary categories. The list contains 5 causes of death not included in the Nolte and $\mathrm{McKee}^{[5]}$ or Tobias and $\mathrm{Yeh}^{[6]}$ lists, i.e. HIV/AIDS, sexually transmitted infections (STIs) excluding 


\section{Table 1. Amenable cause-of-death list}

\begin{tabular}{|c|c|}
\hline NBD cause & ICD-10 code (age restrictions) \\
\hline Tuberculosis & A15 - A19, B90, U51, U52, J90, J94 \\
\hline HIV/AIDS & B20 - B24, B33, B45, B59, C46, D84 \\
\hline STIs, excluding HIV & A50 ( $\geq 1$ month), ${ }^{*}$ A51 - A53, A54 ( $\geq 1$ month), ${ }^{*}$ A55 - A63, N70 - N73, A64 \\
\hline Diarrhoeal diseases & A00 - A09, K52 \\
\hline $\begin{array}{l}\text { Intestinal parasites, other parasitic and vector- } \\
\text { borne diseases }\end{array}$ & A82, A90 - A91, A95, B55 - B57, B65, B67 - B74, B76 - B82, B89 \\
\hline Meningitis and encephalitis & A39, G00 - G03, G05, A83 - A87, G04, G09, G93 \\
\hline Septicaemia & $\mathrm{A} 40-\mathrm{A} 41$ \\
\hline Malaria & B50 - B54 \\
\hline Lower respiratory infections & $\mathrm{J} 09-\mathrm{J} 18, \mathrm{~J} 20-\mathrm{J} 22, \mathrm{P} 23$ (>6 days), ${ }^{*} \mathrm{~J} 86$ \\
\hline Upper respiratory infections & J00 - J06 \\
\hline Otitis media, mastoiditis & H65 - H66 \\
\hline Maternal haemorrhage & O20, O44 - O46, O67, O72 \\
\hline Maternal sepsis & O85 - O86 \\
\hline Hypertension in pregnancy & $\mathrm{O} 10-\mathrm{O} 16$ \\
\hline Obstructed labour & O64 - O66 \\
\hline Abortion & $\mathrm{O} 00-\mathrm{O} 08$ \\
\hline Other maternal & O21 - O43, O47 - O48, O60 - O63, O68 - O71, O73 - O75, O80 - O84, O87 - O92, O94 - O96 \\
\hline Preterm birth complications & P01, P07, P22, P23 (<6 days), ${ }^{*}$ P25 - P28, P29, P52, P61, P77, P80 \\
\hline Birth asphyxia and trauma & P02, P03, P10 - P15, P20 - P21, P24, P50, P90 - P91 \\
\hline $\begin{array}{l}\text { Sepsis and other infectious conditions of } \\
\text { the newborn }\end{array}$ & P35, P36 - P39, P60, A33 - A35 (<1 month), A A0, A54 (<1 month $)^{*}$ \\
\hline $\begin{array}{l}\text { Other non-infectious conditions in } \\
\text { the perinatal period }\end{array}$ & P00, P04, P05, P08, P51, P53 - P59, P62 - P74, P76, P78 - P79, P81 - P89, P93 - P94 \\
\hline Corpus uteri cancer & C54 - C55 \\
\hline Melanoma & $\mathrm{C} 43$ \\
\hline Other skin cancer & $\mathrm{C} 44$ \\
\hline Breast cancer & C50 \\
\hline Cervical cancer & $\mathrm{C} 53$ \\
\hline Testicular cancer & C62 \\
\hline Hodgkin's lymphoma & $\mathrm{C} 81$ \\
\hline Leukaemia & C91 - C95 (<45 years) \\
\hline Bladder cancer & $\mathrm{C} 67$ \\
\hline Thyroid cancer & $\mathrm{C} 73$ \\
\hline Diabetes mellitus & E10 - E14 \\
\hline Epilepsy & G40 - G41 \\
\hline Ischaemic heart disease & I20 - I25 \\
\hline Rheumatic heart disease & I00 - I09 \\
\hline Hypertensive heart disease & I11 \\
\hline Cerebrovascular disease & I60 - I69, G81 \\
\hline Asthma & J45 - J46 (<45 years) \\
\hline Peptic ulcer & K25 - K27 \\
\hline Appendicitis & K35 - K37 \\
\hline Intestinal obstruction and strangulated hernia & K40 - K46, K56 \\
\hline Renal disease & N00 - N08, N10 - N12, N17 - N19, I12, I13 \\
\hline Benign prostatic hypertrophy & N40 \\
\hline Congenital heart anomalies & Q20 - Q28 \\
\hline $\begin{array}{l}\text { Adverse effects of medical and surgical } \\
\text { treatment }\end{array}$ & Y39 - Y66, Y68 - Y84, Y88 \\
\hline
\end{tabular}

HIV, intestinal parasites and other vector diseases, encephalitis and otitis media. HIV/AIDS was added to the local list because of recent evidence indicating that survival is extended by $>5$ years after initiation of antiretroviral (ARV) treatment, regardless of CD4+ cell count. ${ }^{[26]}$ STIs, excluding HIV, intestinal parasites and other vector diseases, encephalitis and otitis media were excluded from the Nolte and $\mathrm{McKe}^{[5]}$ and Tobias and $\mathrm{Yeh}^{[6]}$ lists owing to the scarcity of these causes of death $(<0.1 \%$ of all deaths $)$ in Europe and Australasia. 
Table 2. Age-standardised death rates, deaths and amenable death proportions by province and population group, 2012

\begin{tabular}{llll}
\hline & ASDR & Deaths & Proportion $^{*}$ \\
\hline Province & & & 65.6 \\
$\quad$ KwaZulu-Natal & 958.4 & 83868.8 & 63.2 \\
Free State & 854.1 & 20714.8 & 68.6 \\
Mpumalanga & 851.0 & 28751.2 & 57.4 \\
Eastern Cape & 782.2 & 45220.9 & 64.1 \\
North West & 765.1 & 24565.1 & 58.0 \\
Northern Cape & 706.7 & 7158.5 & 61.9 \\
Limpopo & 641.1 & 28972.6 & 56.8 \\
Gauteng & 536.8 & 58910.7 & 43.9 \\
$\quad$ Western Cape & 378.8 & 20372.4 & 60.2 \\
South Africa & 692.9 & 318534.9 & \\
Population group & & & 64.8 \\
$\quad$ African & 827.6 & 284326.0 & 49.8 \\
Coloured & 436.8 & 17827.1 & 48.7 \\
Asian & 337.9 & 4559.6 & 26.3 \\
White & 186.8 & 11822.1 & \\
ASDR =age-standardised death rate. & & &
\end{tabular}

Table 2 presents ASDRs, total number of deaths and proportions for 2012 - the last year of analysis. In 2012, just $<320000$ deaths in SA were classified as amenable to healthcare, which equates to $60 \%$ of all deaths. The highest number of amenable deaths occurred in KwaZulu-Natal (KZN) Province ( $N=83$ 868), followed by Gauteng Province ( $N=58$ 910).

Using ASDR, there were huge inequalities in amenable mortality between the different provinces and population groups in 2012 (Table 2). KZN had the highest ASDR (958 per 100000 population), 2.5 times higher than the lowest ASDR, which was in the Western Cape (378.8 per 100000 population). The disparities between population groups were even wider, with the African population having an ASDR 4.4 times higher than that of the white population.

Deaths amenable to healthcare accounted for $>50 \%$ of total deaths in all provinces, except in the Western Cape (43.9\%), while Mpumalanga Province had the highest proportion (68.6\%). The white population had a much lower proportion of amenable mortality $(26.3 \%)$ compared with other population groups $(>48 \%)$, while the African population had the highest proportion (64.8\%).

Analysing the trend in amenable mortality between 1997 and 2012, a disparity in ASDR by province in 1997 and population group in 2000 was evident, which widened each subsequent year until 2005 (Figs 1A and B) and narrowed thereafter. The Western Cape had the lowest amenable ASDR over the entire period, while KZN and Free State Province had the highest rates (Fig. 1A). The trend was similar for all provinces, increasing between 1997 and 2006 and decreasing thereafter. The ASDR for SA was 591 per 100000 in 1997, reached a peak (1 074 per 100000 ) in 2005 and decreased thereafter (693 per 100 000) in 2012. The African population had the highest ASDR of all population groups over the period, which was 4.6 times that of the white population in 1997, increasing to 6.5 times in 2005 (Fig. 1B).

When HIV/AIDS as a cause was excluded from the analysis, the ASDR trend decreased for some provinces, such as Eastern Cape Province, the Free State and Mpumalanga, but remained fairly stable over the period for others (Fig. 1C). There was a decreasing trend in ASDR between 2000 and 2012 for all population groups, except for the white population, where there was a minimal change (Fig. 1D).

The mean annual percentage change in ASDR, an indicator of the pace of change, is presented in Fig. 2. It provides a breakdown of the percentage change for SA before and after the HIV/AIDS mortality peak, as well as a breakdown with and without HIV/AIDS. SA had a mean annual increase of $1.36 \%$ in amenable ASDR between 1997 and 2012, but when HIV/AIDS was removed from the analysis, the mean percentage change in ASDR decreased at a rate of 1.12 per year. There was a particularly high average increase of $6.9 \%$ between 1997 and 2006, which was reversed to a decrease of $0.05 \%$ per year when HIV/AIDS was excluded. Between 2007 and 2012, there was an annual average decrease in amenable ASDR of $7 \%$, which reduced to $2.7 \%$ when HIV/AIDS was excluded from the analysis.

An average of 207810 amenable deaths per year could have been averted in SA between 2008 and 2012 if all provinces had the same amenable mortality rate as the Western Cape (Fig. 3A). KZN had the highest excess amenable mortality of all provinces (70 257 deaths). If all population groups had the same amenable mortality rate as the white population, an average of 300000 deaths per year between 2008 and 2012 could have been averted. The African population group accounted for $95 \%$ of all excess deaths. Males and females accounted for similar numbers of excess amenable deaths across provinces and population groups (Fig. 3B).

The amenable ASDRs from SA were compared with those of countries assessed in the Eurostat working group and Gay et al. ${ }^{[11]}$ studies by using the lists of amenable conditions and standard populations from these studies (Figs $4 \mathrm{~A}$ and $\mathrm{B}$ ). ${ }^{[11,20]}$ There were huge disparities in ASDRs between SA and the other countries. The ASDR for SA was 2.6 times higher than that of the Eurostat working group country with the highest ASDR and 11.5 times higher than that of the country with the lowest ASDR for 2012 (Fig. 4A). The ASDR for SA was 2.2 times higher than that of the worst-performing OECD country, using the Tobias and $\mathrm{Yeh}^{[6]}$ list, and 1.8 times higher than that of the worst-performing country using the Nolte and McKee ${ }^{[5]}$ list.

\section{Discussion}

To the best of our knowledge, this study of the epidemiology of amenable mortality in SA is the first to be reported. The local amenable cause-of-death list contains more causes of death than the frequently used lists of Nolte and $\mathrm{McKe}^{[5]}$ and Tobias and Yeh, ${ }^{[6]}$ reflecting the quadruple burden of disease in SA, with high rates of infectious and non-infectious diseases. The local list includes causes such as HIV/AIDS, STIs excluding HIV/AIDS, and intestinal parasites and vector diseases, which are not present in the other lists. 


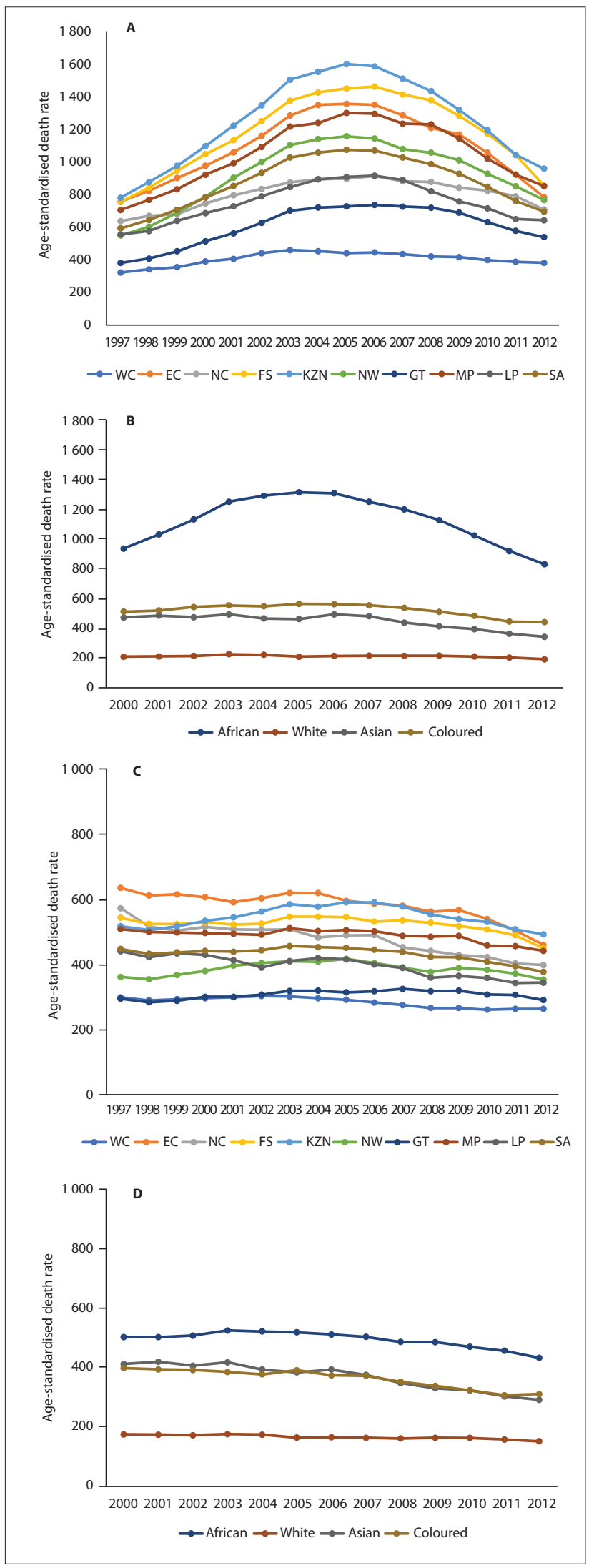

Fig. 1. Trend in age-standardised amenable mortality by: (A) province between 1997 and 2012; (B) population group between 2000 and 2012; (C) province, excluding HIV/AIDS; and (D) population group, excluding HIV/AIDS. $($ WC $=$ Western Cape; EC = Eastern Cape; NC = Northern Cape; $F S=$ Free State; KZN = KwaZulu-Natal; $N W=$ North West $; G T=$ Gauteng; $M P=$ Mpumalanga; $L P=$ Limpopo; $S A=$ South Africa. )

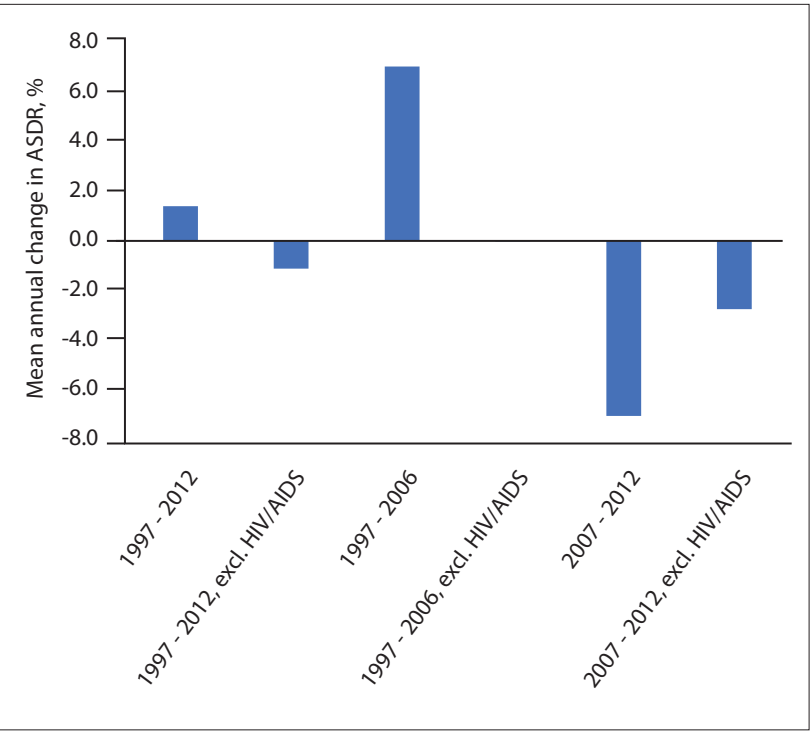

Fig. 2. Comparison of mean annual percentage change in ASDR in South Africa across different time periods, with and excluding HIV/AIDS. (ASDR = age-standardised death rate; excl. = excluding.)

HIV/AIDS was not included in amenable cause-of-death lists until recently, when the Eurostat ${ }^{[20]}$ study included it in both amenable and preventable cause lists. Triple-therapy ARVs have been available since the mid-1990s, but only recently has evidence emerged that the life expectancy of individuals with HIV/AIDS after initiation of ARVs is $\sim 80 \%$ of the life expectancy of people without HIV/ AIDS. ${ }^{[26]}$

The high proportion and ASDRs indicate an ineffective healthcare system overwhelmed by a high burden of disease. The HIV/AIDS epidemic placed immense strain on the health system and is likely to have detracted healthcare supply from other health conditions. People with HIV/AIDS occupied a large proportion of available hospital beds, and the prevalence of HIV/AIDS in the health workforce was estimated at $16-20 \%$ in $2006 .{ }^{[27]}$ This could explain the relatively low average annual decline of $1.12 \%$ in amenable mortality rates compared with those of other countries when HIV/AIDS is excluded from the analyses. For instance, in the Gay et al. ${ }^{[11]}$ study, the average annual decline in amenable ASDR was 3.5\% between 1997 and 2007, while countries with an above-average amenable ASDR in 1997 showed a higher rate of decline. After the peak in HIV/ AIDS mortality in 2006, there does appear to be an improvement in the performance of the health system, with rates of amenable causes steadily decreasing at an average annual rate of $7 \%$, and 2.7\% when HIV/AIDS is excluded from the analysis. The decrease in amenable mortality after 2006 when ARVs were introduced into the healthcare system in SA is due largely to HIV/AIDS. The rapidly reducing HIV/AIDS mortality probably also had an indirect positive impact on the quality of care for other amenable causes during this period. Furthermore, there have been some government initiatives to reduce the burden of disease following a change in health sector leadership in 2009, as described by Mayosi et al., ${ }^{[28]}$ which may have contributed to the improved amenable mortality rates.

There were huge disparities in amenable mortality between provinces and population groups, which did not attenuate much over a 15-year period. To reduce the disparity, causes other than HIV/AIDS also need to decline. Although the pace of decline in amenable mortality excluding HIV/AIDS improved after 2006 in most provinces and population groups, it did not occur in a pattern that would reduce the disparities between provinces and population 


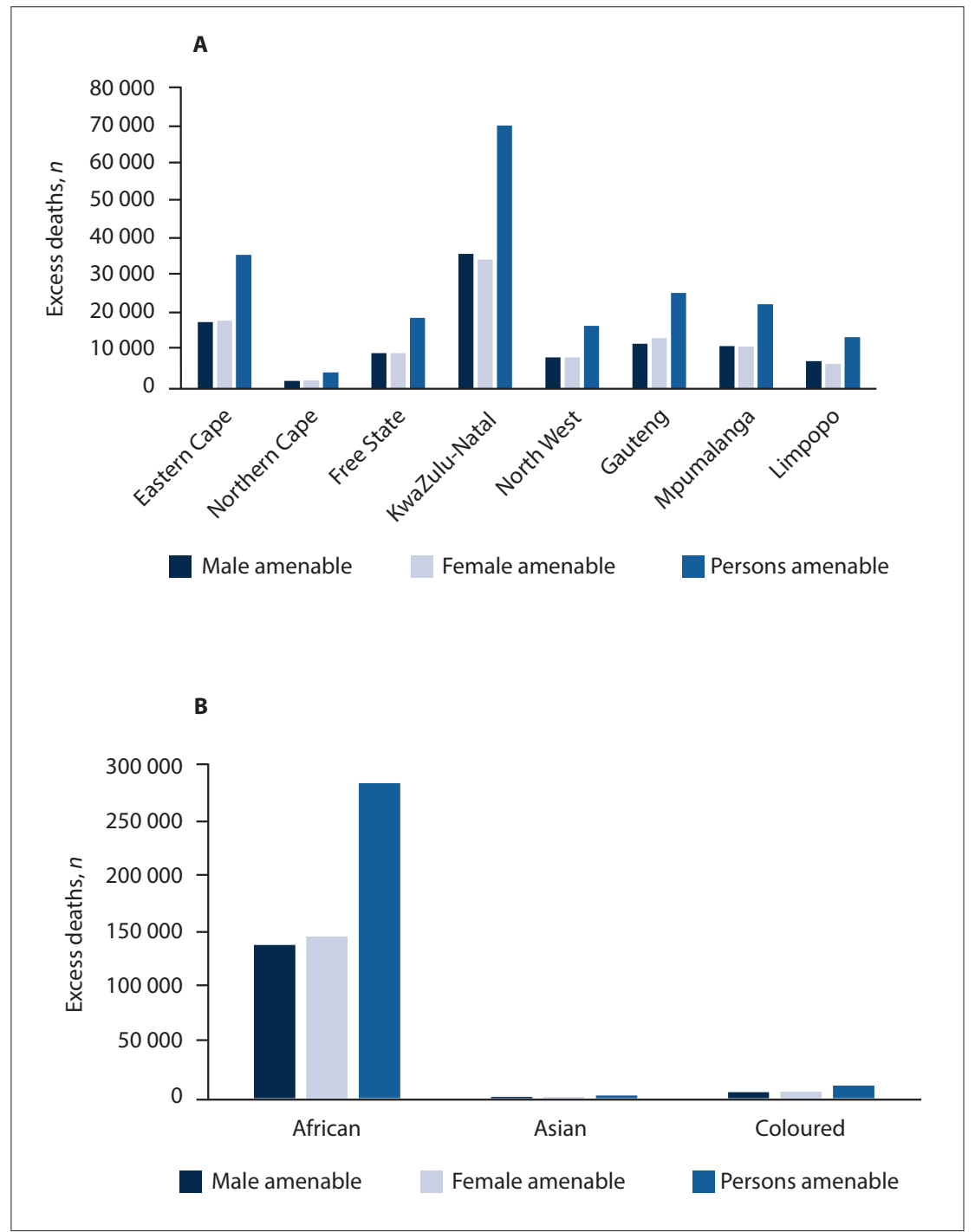

Fig. 3. Average excess mortality between 2008 and 2012 by: (A) province; (B) population group.

groups over time. For instance, in 1997, KZN had the fourth-largest amenable mortality ASDR excluding HIV/AIDS, but the mean annual decrease was the third-lowest for all provinces. In the same year, Limpopo had the third-lowest amenable mortality ASDR, but the third-lowest mean annual decrease.

The high rates of amenable mortality could be related to the inefficient distribution of public and private care, with an estimated $85 \%$ of the population dependant on public health services while the distribution of healthcare is skewed towards private care. ${ }^{[29]}$ Inequitable healthcare financing probably also played a role in the disparity in amenable mortality between the different provinces and population groups. Health financing has been shown to be inversely proportional to need at district and sub-district level. ${ }^{[30,31]}$ This disparity in healthcare financing is perpetuated by a greater allocation of funding to provinces with a higher number among whites from the start, but could also indicate that access to quality healthcare for whites has decreased over the period or that quality of healthcare for other population groups has improved.

The excess death approach is a useful measure, as it calculates the number of amenable deaths that could have been prevented if health system performance was at the same level as the best-performing province or population group. The largest number of excess amenable deaths occurred in KZN and in the African population, and gives an indication of the impact that can be made by reducing amenable mortality in the most vulnerable groups.

The pending NHI is intended to supply essential healthcare to all legal residents and could potentially aid in reducing amenable mortality levels, as well as the disparities in amenable mortality between provinces and population groups. Universal healthcare has been effective in reducing inequities in amenable mortality, e.g. in Canada, the difference in amenable mortality decreased by $60-78 \%$ between the richest and poorest neighbourhoods between 1971 and 1996 after the introduction of universal healthcare, while similar findings have been shown in the UK and Australia. ${ }^{[15-17]}$

The benchmarking of SA against countries analysed in the Gay et al. ${ }^{[1]}$ and Eurostat ${ }^{[20]}$ studies reveals a health system that is performing poorly. However, the comparison might be unfair, as these are mostly high-income countries. SA's relatively poor health performance was also shown in a study that compared Brazil, Russia, India, China and SA with the economic peers of each country. SA performed the worst over a 20-year period. ${ }^{[35]}$ This study showed declining health performance in SA, with excess years of life lost (YLLs), calculated as the difference between actual YLLs and YLLs in the best-performing country, which increased sharply between 1990 and 2011.

There are some limitations to the use of amenable mortality as an indicator of health system performance that have been documented elsewhere. ${ }^{[36,37]}$ The measure does not account for factors outside the scope of healthcare that could influence amenable mortality, such as socioeconomic circumstances. It does not allow for an assessment of morbidity or provide for plans to improve the health system. The list of amenable causes should be regularly reviewed to include those that have become amenable owing to advances in medical technology.

Some limitations specific to this study are that the NBD data are based on statistical 


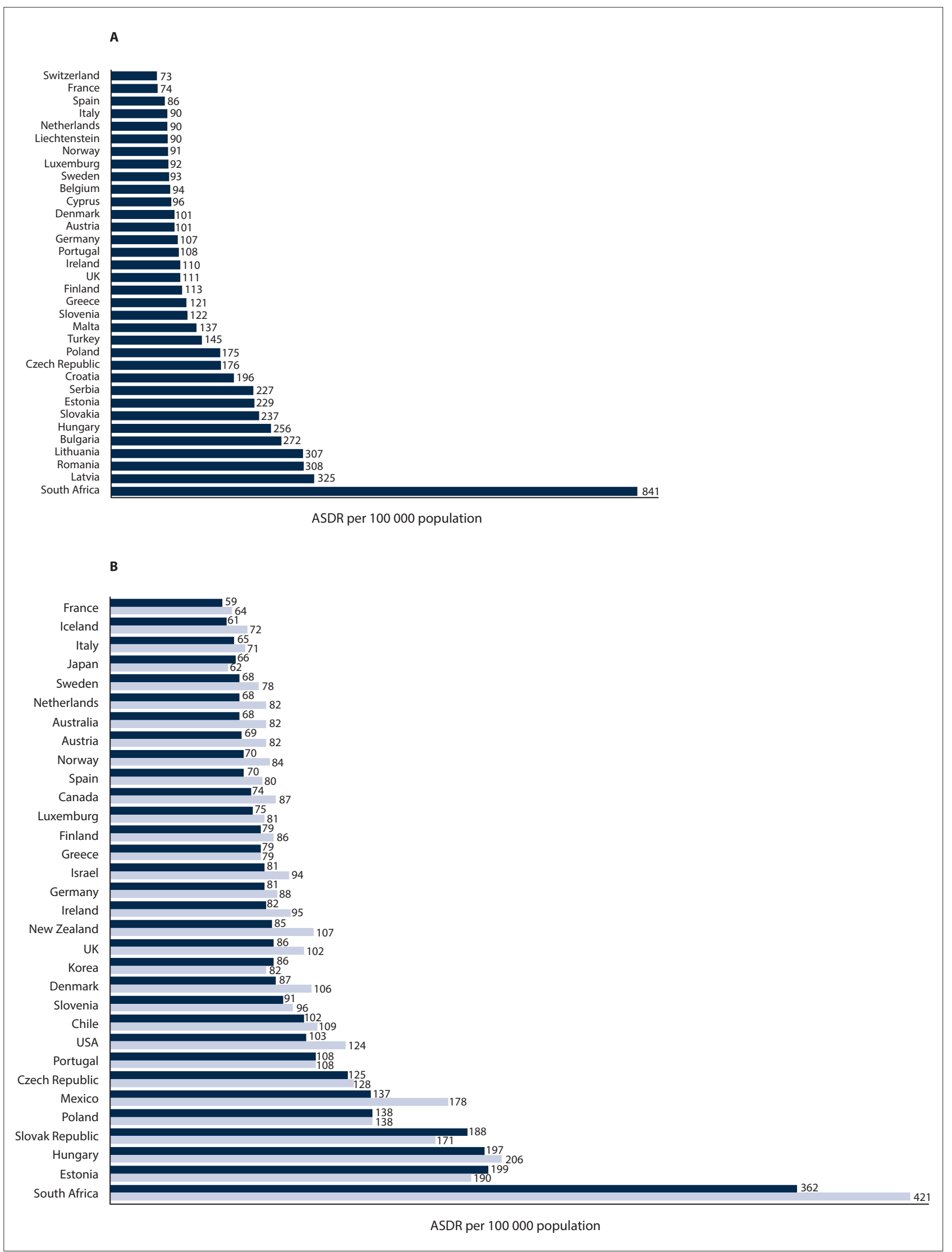

Fig. 4. Comparison of amenable ASDR for South Africa with: (A) Eurostat countries; ${ }^{[2]}$ and (B) Organisation for Economic Co-operation and Development countries. ${ }^{[1]]}\left(A S D R=\right.$ age-standardised death rate; $(B)$ dark-blue bar = estimates using Nolte and McKee ${ }^{[5]}$ amenable list; light-blue bar = estimates using Tobias and Yeh ${ }^{[6]}$ amenable list.) 
modelling approaches using various assumptions. However, the estimates have been validated against other data sources and are an improvement on the StatsSA data. HIV/AIDS was categorised as an amenable cause of death even though ARVs were only introduced to the SA health system in 2006. However, SA chose not to provide ARVs before 2006 and health system performance should be assessed on the best-available healthcare. We were unable to do a comparison with the global list of countries assessed in the Lancet Global Commission on High Quality Health Systems study ${ }^{[19]}$ because they reported on 2016 mortality data, which are not represented in the NBD data; they did not report age-standardised rates.

\section{Conclusions}

Amenable mortality could be used as an indicator to monitor and evaluate the performance of SA's health system. This is especially relevant in light of the pending NHI. In combination with other indicators, such as resource allocation and service coverage, amenable mortality could provide important insight into the success of $\mathrm{NHI}$ reforms. It could track progress in reducing geographical and racial inequities and identify priority areas of the health system that require improvement. Ideally, amenable mortality should be routinely monitored at district and sub-district levels, each of which may require a different set of targets and benchmarks. At facility level, it could be used as a tool to investigate and conduct reviews of each amenable death. As a globally recognised metric, it is important to track progress of overall amenable mortality levels at national level compared with those of other countries. The ASDR level and annual percentage change in amenable mortality should be used together to assess performance, while the list of amenable causes should be reviewed on a continuous basis. Ultimately, there is a need to develop the capability to conduct and integrate amenable mortality assessments into the NHI system.

\section{Declaration. None.}

Acknowledgements. We are grateful to the public health experts and clinicians who participated in the round-table meeting on the amenable mortality list at the 2nd Western Cape Research Day hosted at Lentegeur Hospital, Cape Town, and the disease-specific experts who provided input on specific causes of death. We are also grateful to the NBD study team for allowing access to the 2nd SA NBD study estimates.

Author contributions. IN, PG, HS, DB conceived and designed the study; IN analysed and prepared the data for analysis; IN, PG, HS, DB contributed to the writing of the manuscript; IN, PG, HS, DB agreed regarding the manuscript results and conclusions.

Funding. Research reported in this publication was supported by the SA Medical Research Council (SAMRC). Its contents are solely the responsibility of the authors and do not necessarily represent the official views of the SAMRC.

Conflicts of interest. None.

1. Pillay-van Wyk V, Msemburi W, Laubscher R, et al. Mortality trends and differentials in South Africa from 1997 to 2012: Second National Burden of Disease Study. Lancet Global Health 2016;4(9):e642-e653. https://doi.org/10.1016/S2214-109X(16)30113-9

2. Inter-Agency and Expert Group on SDG Indicators. Report on the inter-agency and expert group on sustainable development goal indicators (E/CN.3/2017/2), Annex III 2017. https://unstats.un.org/ on sustainable development goal indicators (E/CN.3/2017/2), Annex III 2017. https://un
unsd/statcom/48th-session/documents/2017-2-IAEG-SDGs-E.pdf (accessed 1 July 2019).

3. Kruk ME, Gage AD, Arsenault C, et al. High-quality health systems in the sustainable development goals era: Time for a revolution. Lancet Global Health 2018;6(11):el196-e1252. https://doi.org/ 10.1016/S2214-109X(18)30386-3
4. Rutstein DD, Berenberg W, Chalmers TC, Child CGD, Fishman AP, Perrin EB. Measuring the quality of medical care. A clinical method. N Engl J Med 1976;294(11):582-588. https://doi.org/10.1056/ NEJM197603112941104

5. Nolte E, McKee M. Measuring the health of nations: Updating an earlier analysis. Health Affairs 2008;27(1):58-71. https://doi.org/10.1377/hlthaff.27.1.58

6. Tobias M, Yeh L. How much does health care contribute to health gain and to health inequality? Trends in amenable mortality in New Zealand 1981 - 2004. Aust N Z Public Health 2009;33(1):70-78. https:// doi.org/10.1111/j.1753-6405.2009.00342.x

7. Holland WW. European community atlas of 'avoidable death'. Commission of the European Communities Health Services Research Series No. 3. Oxford: Oxford University Press, 1988.

8. Ramkissoon FE. Commentary on 'in amenable mortality - deaths avoidable through health care progress in the US lags that of three European countries'. Ann Med Surg 2013;2(1):5-7. https://doi. org/10.1016/S2049-0801(13)70018-9

9. Charlton JR, Hartley RM, Silver R, Holland WW. Geographical variation in mortality from conditions amenable to medical intervention in England and Wales. Lancet 1983;26(8326):691-696. https://doi. org/10.1016/s0140-6736(83)91981-5

10. Tobias M, Jackson G. Avoidable mortality in New Zealand, 1981 - 97. Aust N Z J Public Health Econ 2001;25(1):12-20. https://doi.org/10.1111/j.1467-842X.2001.tb00543.x

11. Gay JG, Paris V, Devaux M, de Looper M. Mortality Amenable to Health Care in 31 OECD Countries: Estimates and Methodological Issues. OECD Health Working Papers No. 55. Paris: OECD, 2011.

12. Schoenbaum SC, Schoen C, Nicholson JL, Cantor JC. Mortality amenable to health care in the United States: The roles of demographics and health systems performance. J Public Health Policy 2011;32(4):407-429. https://doi.org/10.1057/jphp.2011.42

13. Hoffmann R, Borsboom G, Saez M, et al. Social differences in avoidable mortality between small areas of 15 European cities: An ecological study. Int J Health Geographics 2014;13:8. https://doi. org/10.1186/1476-072X-13-8

14. Park J, Tjepkema M, Goedhuis N, Pennock J. Avoidable mortality among first nations adults in Canada: A cohort analysis. Health Reports 2015;26(8):10-16

15. James PD, Wilkins R, Detsky AS, Tugwell P, Manuel DG. Avoidable mortality by neighbourhood income in Canada: 25 years after the establishment of universal health insurance. J Epidemiol Comm Health 2007;61(4):287-296. https://doi.org/10.1136/jech.2006.047092

16. Korda RJ, Butler JRG, Clements MS, Kunitz SJ. Differential impacts of health care in Australia: Trend analysis of socioeconomic inequalities in avoidable mortality. Int J Epidemiol 2007;36(1):157-165. https://doi.org/10.1093/ije/dyl282

17. Desai M, Nolte E, Karanikolos M, Khoshaba B, McKee M. Measuring NHS performance 1990 2009 using amenable mortality: Interpret with care. J R Soc Med 2011;104(9):370-379. https://doi. org/10.1258/jrsm.2011.110120

18. Department of Health. The NHS Outcomes Framework 2012/13. London: DoH, 2010.

19. Kruk ME, Gage AD, Joseph NT, Danaei G, García-Saisó S, Salomon JA. Mortality due to low-quality health systems in the universal health coverage era: A systematic analysis of amenable deaths in 137 countries. Lancet 2018;392(10160):2203-2212. http: // doi.org/10.1016/S0140-6736(18)31668-4

20. Eurostat. Amenable and preventable deaths statistics: Eurostat. 2016. http://ec.europa.eu/eurostat/ statistics-explained/index.php/Amenable_and_preventable_deaths_statistics (accessed 19 June 2019).

21. Pillay-van Wyk V, Laubscher R, Msemburi W, et al. Second South African National Burden of Disease Study: Data Cleaning, Validation and SA NBD List. Cape Town: Burden of Disease Research Unit, South African Medical Research Council, 2014.

22. Lavergne MR, McGrail K. What, if anything, does amenable mortality tell us about regional health system performance? Healthcare Policy 2013;8(3):79-91.

23. Quercioli L, Messina G, Basu S, McKee M, Nante N, Stuckler D. The effect of healthcare delivery privatisation on avoidable mortality: Longitudinal cross-regional results from Italy, 1993 - 2003. J Epidemiol Comm Health 2013;67(2):132-138. https://doi.org/10.1136/jech-2011-200640

24. Dorrington RE. Alternative South African Mid-year Estimates, 2013. Cape Town: Centre for Actuarial Dorrington RE. Alternative South African
Research, University of Cape Town, 2013.

25. Ahmad OB, Boschi-Pinto C, Lopez AD, Murray CJL, Lozano R, Inoue M. Age standardization of rates: A new WHO standard. Geneva: World Health Organization, 2001

26. Johnson LF, Mossong J, Dorrington RE, et al. Life expectancies of South African adults starting antiretroviral treatment: Collaborative analysis of cohort studies. PLoS Med 2013;10(4):e1001418. https://doi.org/10.1371/journal.pmed.1001418

27. Joint United Nations Programme on HIV/AIDS. Report on the Global AIDS Epidemic. A UNAIDS 10th Anniversary Special Edition. Geneva: UNAIDS, 2006

28. Mayosi BM, Lawn JE, van Niekerk A, et al. Health in South Africa: Changes and challenges since 2009. Lancet 2012;380(9858):2029-2043. https://doi.org/10.1016/S0140-6736(12)61814-5

29. McIntyre D, Thiede M, Nkosi M, et al. A Critical Analysis of the Current South African Health System. Cape Town: Health Economics Unit, University of Cape Town and Centre for Health Policy, University of the Witwatersrand, 2007.

30. Thomas S, Mbatsha S, Muirhead D, Okorafor O. Primary health care financing and need across health districts in South Africa. 2004. http://citeseerx.ist.psu.edu/viewdoc/download?doi=10.1.1.541.9081\&r ep=rep 1\&type=pdf (accessed 19 June 2019).

31. Scott V, Stern R, Sanders D, Reagon G, Mathews V. Research to action to address inequities: The experience of the Cape Town equity gauge. Int J Equity Health 2008; 7(6). https://doi.org/10.1186/14759276-7-6

32. Stuckler D, Basu S, McKee M. Health care capacity and allocations among South Africa's provinces: Infrastructure-inequality traps after the end of apartheid. Am J Public Health 2011;101(1):165-172. https://doi.org/10.2105/AJPH.2009.184895

33. Massyn N, Day C, Barron P, et al. District Health Barometer 2011/12. Durban: Health Systems Trust, 2013.

34. Harris B, Goudge J, Ataguba JE, et al. Inequities in access to health care in South Africa. J Public Health Policy 2011;32(Suppl 1):S102-S123. https://doi.org/10.1057/jphp.2011.35

35. Petrie D, Tang KK. Relative health performance in BRICS over the past 20 years: The winners and losers. Bull Health Organ 2014;92:396-404

36. Nolte E, McKee M. Does Health Care Save Lives? Avoidable Mortality Revisited. London: Nuffield Trust, 2004.

37. Page A, Tobias M, Glover J, Wright C, Hetzel D, Fisher E. Australian and New Zealand Atlas of Avoidable Mortality. Adelaide: PHIDU, University of Adelaide, 2006.

Accepted 11 February 2019. 